\title{
Non-Contact Axial Work piece Diameter Size Measuring System
}

\author{
Hong Zheng ${ }^{\text {a }}$, Qi Lu ${ }^{\mathrm{b}}$ \\ University of Technology, Jinan 250353, China \\ azhh@qlu.edu.cn, b267353557@qq.com
}

Keywords: laser sensor, automatic scan, servo motor.

\begin{abstract}
This article designs a non-contact axial work piece diameter size measuring system. It uses high accuracy laser sensor and precision linear module. This paper introduced working principle of the system, mechanical structure and measuring range. The characteristics include non-contact, high precision, automatic scanning and measuring range can be adjusted easily. The system is simple to adjust, easy to operate, especially suitable for plant operations or inspection as an important way of enterprise internal quality control. In addition, non-contact measurement is also significantly enhance the adaptability to environment and suitable for continuous measurement of work piece in production line.
\end{abstract}

\section{Introduction}

In the mechanical parts production process, outer diameter of parts measurement plays an important role. Quickly and accurately measure the main parameters of components can short the production cycle, realize automatic production and improve production efficiency ${ }^{[1]}$.

Non-contact measuring system based on optical methods, it breaks people's traditional thinking mode which is contact directly with objects when measuring objects. It has important theoretical significance ${ }^{[2]}$. Manual inspection can easily cause visual fatigue, so detection efficiency is low and precision is low and range of applications is narrow ${ }^{[3] .}$

\section{The working principle of measuring system}

Figure 1-1 is a tested part which shows that the dimension measure is so many. Some sizes are measured difficulty even can't be measured directly. And traditional measurement wastes time, so non-contact measuring system was designed.

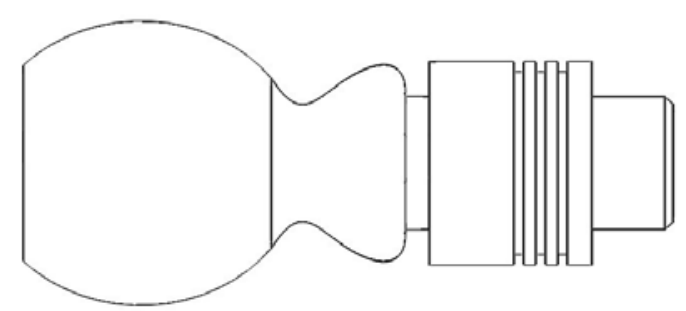

Fig.1-1 a tested part

Automatic measurement system uses servo motor to drive precision linear module and slider orientation. Two sets of CCD laser sensors are installed on the slider. Laser sensor scans outer outline of the workpiece continuously when move to the desired location. The measured data are processed by IPC. It calculates the main size parameters of outside diameter. It can real-time display measurement results, save and print the test data.

If the size is beyond the measurement scope of the two groups of laser sensor, the linear module can be adjusted the distance of installation. It can enlarge the measuring range of system and meet the needs of the measurement. The working principle of measuring system is shown in figure 1-2: 


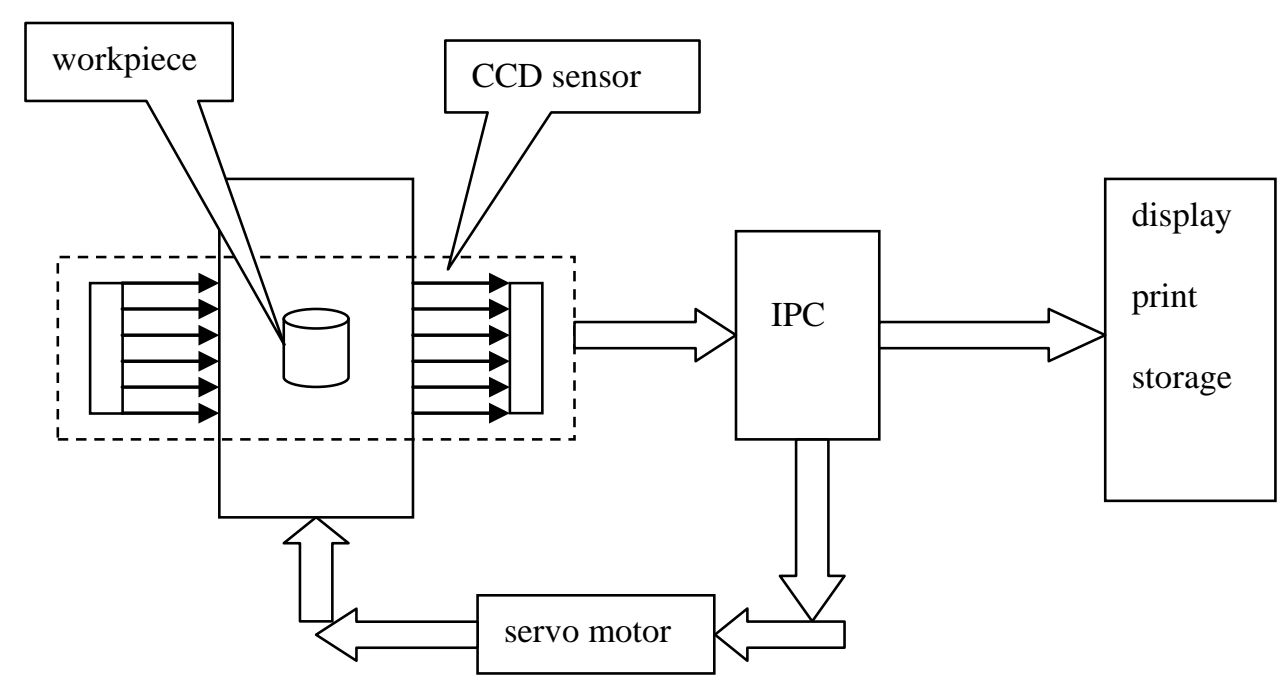

Fig. 1-2 Working principle of measurement system

\section{Measuring system}

Mechanical structure. Mechanical structure adopts servo motor can control the speed and position accurately. It is low noise and no step running conditions. In closed-loop system, the signal is transmitted to the system at any time, and movement is adjusted by feedback signal of the system at the same time. Precision linear motion module runs stability, and has high positioning accuracy and no return clearance. These are to further improve the accuracy of the motion system. Two-dimensional figure of the mechanical structure is shown in the Fig.2-1:

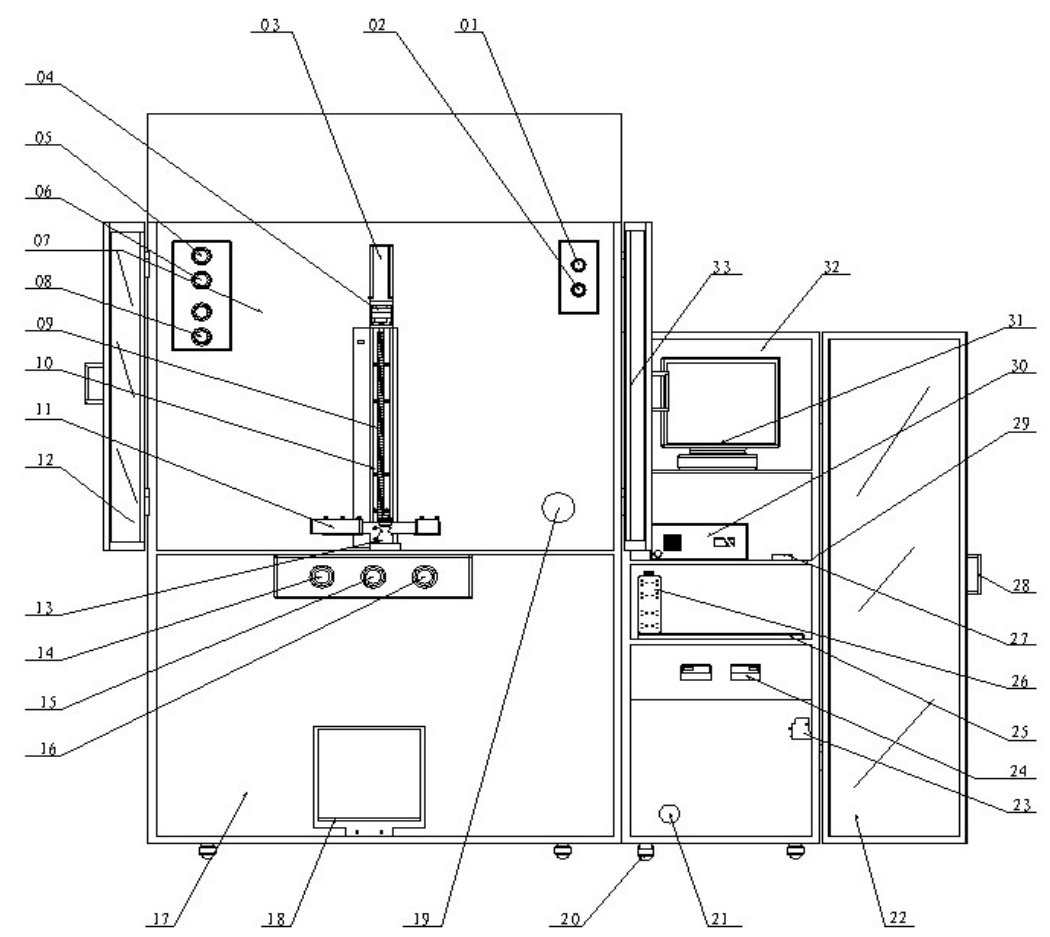

Fig.2-1 Two-dimensional figure of the mechanical structure

01. Contact button 02.cut-off button 03.servo motor 04. Coupler 05.power light 06.alarm indicator lamp 07.qualified indicator light 08.unqualified indicator light 09.ball screw 10.motion components 11. CCD sensor 12.left hand door 13.part 14.reset button 15.scram button 16.activate button 17.big cabinet 18.foot rest 19.line hole(big cabinet) 20. Truckle 21.line hole(distribution box) 22.mirror door 
23.button 24.digital display 25.Keyboard 26.Connector 27.Mouse 28.Handle 29.side plate 30. Industrial control computer 31.Display 32.distribution box 33.right side door

Measuring system of $3 \mathrm{~d}$ model as shown in figure 2-2:
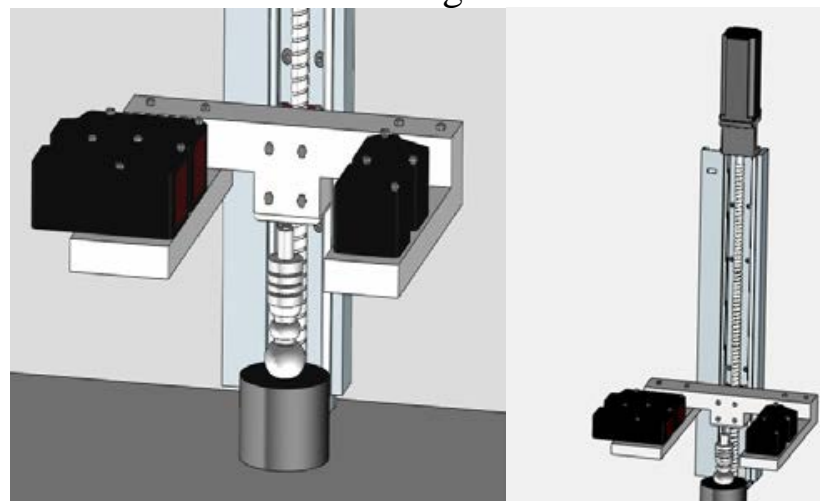

Fig.2-2 3 d model of measurement system

Optical system. CCD laser sensor ${ }^{[4]}$ uses high-precision light through laser sensor IG-028 of Keyence. Sensor optical parts have a transmitter and a receiver. Laser diode can emit red laser which becomes collimated beam through lens. When the beam is sheltered by the object that forms corresponding shaded area at the receiving end, the receiver scans and calculates the shadow zone length, and size of the measured object can be concluded in the end.

Motion control system. Control system can make from the motor control card, motor drive and servo motor. The motor control card is 4 axis universal PCI structure step by step/pulse type. Motor and drive are the AC servo motor system of Panasonic ${ }^{[5]}$. It has $2500 \mathrm{p} / \mathrm{r}$ incremental encoder and cooperated with drive resolution can be achieved 1/10000.Because the screw lead of system is $10 \mathrm{~mm}$, so the system control precision can reach $1 \mu \mathrm{m}$.

Software system. Software part, Monitor and Control Generated System is used in the non-contact axial workpiece diameter size measuring system. MCGS is the configurable software system based on the Windows. It is used for rapid construction and generating the PC monitoring system. It can finish field data acquisition and monitoring, the front-end data processing and control. With the field data collection and processing, it can provide the solution of actual engineering problems to users with various ways such as animation display, alarm processing, process control and report output in the automation filed.

Measuring range of system. The measuring range of a single set of laser sensor is restricted by the parallel laser beam width. In the non-contact measuring system, the type IG028 of Keyence is used, the measuring range is 0 to $28 \mathrm{~mm}$. As shown in figure $3-1$ :

In the non-contact measuring system, the combined use of two sets of laser sensor makes the measuring range not only confined to the parallel laser beam width. As shown in figure 3-2:

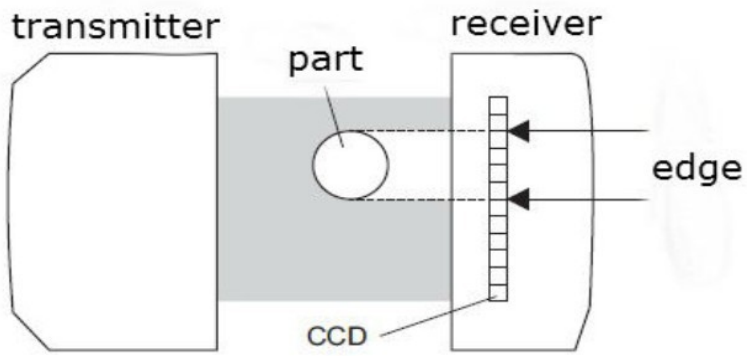

Fig.3-1 measurement of a single set senor

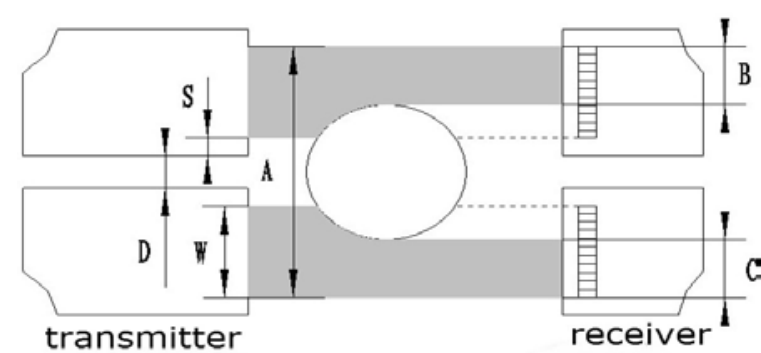

Fig.3-2 combined measurement

Two sets of sensors can respectively through serial port 1 and serial port 2 put $B$ and $C$ to the computer. A can be measured after sensors were installed. The measurement A will be used as fixed parameters directly in the program.

In the measuring instrument, the width of parallel laser beams is: $\mathrm{W}=28 \mathrm{~mm}$.

The mounting distance between two sets of sensors is: $\mathrm{D}=15.8 \mathrm{~mm}$ (the distance can be adjusted.) The distance between the side of parallel laser beam and side of sensor is: $\mathrm{S}=11.6 \mathrm{~mm}$. So,

$A=D+2 \times(W+S)$ 
The diameter work piece in the program:

$$
\phi=A-(B+C)-\mu
$$

$\mu$ : system error. After combined using with two sets of senor, the measuring range is $0 \mathrm{~mm}$ to $100 \mathrm{~mm}$. Of course, Actual measurement range can also continue to expand, according to the distance of installation between two sets of sensors.

\section{Conclusions}

In the modern production, such as automotive engine and aerospace parts, their component parts must have high accuracy in order to reduce noise and to prevent the pollution of the environment and save energy consumption. So the high precision measurement is very important in the early period of the workpiece, but the non-contact axial workpiece diameter size measuring system can solve these problems.

Measurement precision of the non-contact axial workpiece diameter size measuring system is high. It can suitable for batch automation measurement of workpiece. It can improve the efficiency of measurement, reduce human error and record all test data in automatic production line. The data can provides a good basis for analysis process and improve the processing technology.

All in all, with the development of optical technology, measurement technology must realize high precision, high speed and high-efficiency. Therefore non-contact measurement technology will become an important development direction of precision measurement technology.

\section{References}

[1] Ni Shoujun, Jiang Wei, The positioning error analysis of outside diameter of bearing on-line measurement system [J], Bearing, 2005, 48(11):33-36.

[2] Zhou Zhiming, High precision measurement of auto parts dimension based on the optical methods [D], Tongji university, Shang hai,2004.

[3] Lei Liang yu, Zhou Xiaojun, Pan Mingqing, Machine Vision System for Inner and Outer Diameters Inspection of a Bearing [J]. Journal of agricultural machinery, (2005) 131-134.

[4] Liu Zhongpo, Du Baojiang, Automatic measurement system of parts size based on machine vision [J], Mechanical manufacture and automation, (2006) 47-49.

[5] Shu Zhibing. AC servo control system [M], Tsinghua University Press, Beijing, (2006)126-152. 